\title{
Bulgarien auf dem Weg zu demokratischen zivil-militärischen Beziehungen
}

\author{
Stanimira Tchoubatarova*
}

\begin{abstract}
Like all former Warsaw Pact states the Bulgarian forces also face the task of reducing and restructuring. With this, their integration into the democratic state under the rule of law plays an important role in this process. This article analyses specific Bulgarian problems and possible approaches to solve them.
\end{abstract}

Keywords: Demokratische Kontrolle der Streitkräfte; Streitkräftetransformation in Bulgarien

\section{Demokratische Kontrolle der Streitkräfte: Theorie und Praxis}

$\mathrm{I}$ n der Theorie mag die demokratische Kontrolle der Streitkräfte ganz einfach erscheinen: Gewählte Vertreter treffen zusammen mit der Regierung alle wichtigen Entscheidungen zur Verteidigungspolitik und zu den Streitkräften eines Landes. Der Kern demokratischer zivil-militärischer Beziehungen kann daher verstanden werden als die politische Kontrolle des Militärs durch die demokratisch gewählten Organe des Staates. ${ }^{1}$ In einer Demokratie sollte dies zumindest der Fall sein.

In der Praxis ist es leider nicht so einfach. Die Struktur der Streitkräfte, ihre strenge, auf Gehorsam ruhende Hierarchie, die Wehrpflicht sowie konservative Traditionen scheinen den demokratischen Werten in gewissem Maße entgegenzustehen. Angesichts der entscheidenden Aufgabe der Streitkräfte - die Landesverteidigung - ist die Gesellschaft normalerweise bereit, nicht nur ein gewisses Demokratiedefizit im Militär zu akzeptieren, sondern auch seinen außergewöhnlichen und in einigen Fällen privilegierten Status unkritisch hinzunehmen. Bereits Platon hatte in seiner Politeia die Frage aufgeworfen, die seitdem immer wieder gestellt wurde: »Wer bewacht die Wächter?«

Der Daseinszweck der demokratischen Kontrolle besteht darin, Sicherheits- und Verteidigungsangelegenheiten den größeren Zielen einer Nation unterzuordnen und nicht umgekehrt. Der Zweck der Streitkräfte sollte sein, der Gesellschaft, aus der sie hervorgegangen sind, zu dienen und die gesellschaftlichen Werte zu verteidigen, anstatt sie zu dominieren. Aus diesem Grund ist es notwendig, relevante Verfahren und Hilfsmittel für die demokratische Kontrolle der Streitkräfte zu entwickeln.

Um die demokratische Kontrolle der Streitkräfte wirksam zu gestalten, müssen mindestens drei Kriterien erfüllt sein:

1. Ein funktionierender demokratischer Rahmen des Staates, einschließlich der Rechtsstaatlichkeit, der Gewaltenteilung,

* Stanimira Tchoubatarova ist Rechtsberaterin im bulgarischen Verteidigungsministerium. Sie war Baudissin-Fellow am Institut für Friedensforschung und Sicherheitspolitik an der Universität Hamburg (IFSH).

1 Andrew Cottey, Demokratische Kontrolle von Streitkräften im OSZE-Gebiet: Probleme und Herausforderungen, OSZE-Jahrbuch 2001, Hrsg. Institut für Friedensforschung und Sicherheitspolitik an der Universität Hamburg (IFSH), Nomos Verlagsgesellschaft Baden-Baden 2002, S. 308 der Achtung der Menschen- und Bürgerrechte, der Grundfreiheiten und freier, demokratischer Wahlen.

2. Die eindeutige Unterordnung der Streitkräfte unter die Regierung und ihre Pflicht, sich dem Parlament gegenüber zu rechtfertigen.

3. Die politische Neutralität der Streitkräfte und ihr Verzicht auf jegliche Einmischung in innere politische Angelegenheiten. $^{2}$

Im Folgenden sollen wichtige Elemente und Voraussetzungen der Kontrolle der Streitkräfte in Bulgarien betrachtet werden. Ausgehend vom gesetzlichen Rahmen, der parlamentarischen Überwachung und der Kontrolle durch die Exekutive wird das Verhältnis von Militär und Gesellschaft erläutert. Anschließend wird die Ausbildungsproblematik sowie der internationale Kontext angesprochen, bevor am Ende auf den Strukturwandel der bulgarischen Armee und die Perspektiven von demokratischen Kontrollmöglichkeiten eingegangen wird.

\section{Der gesetzliche Rahmen}

Es ist klar, dass die Fragen der demokratischen Kontrolle durch kein Gesetz allein gelöst werden können. Erst nach der Verabschiedung der neuen Gesetze wird sich herausstellen, ob die für die demokratische Kontrolle notwendigen Faktoren wirksam werden. Dazu zählen unter anderem das Bildungsniveau der Soldaten, die Sachkenntnis der Zivilisten im Parlament und in der Exekutive und das in der Öffentlichkeit vorhandene allgemeine Verständnis. Erst unter diesen Vorraussetzungen kann demokratische Kontrolle funktionieren. Im Idealfall sollte versucht werden, überholte Verhaltensweisen auf allen Ebenen (d.h. Öffentlichkeit, Bürokratie, Militär) gleichzeitig zu ändern, damit ein Neuanfang gewährleistet ist. Die demokratische Kontrolle zählt zu den entscheidendsten Faktoren für das Funktionieren der Zivilgesellschaft (ähnlich wie die Pressefreiheit) und sollte deshalb mit Fingerspitzengefühl umgesetzt werden, damit das reibungslose Gelingen gewährleistet werden kann.

Die Rechtsstaatlichkeit ist eine wesentliche Voraussetzung für die Umsetzung der demokratischen Kontrolle. Es liegt auf der Hand, dass es vor der Verabschiedung der bulgarischen Verfassung von 1991 keinerlei rechtliche Grundlage für die Regelung

2 Vgl. Samuel P. Huntington, The Soldier and the State, Cambridge 1957, S. $80 \mathrm{f}$ 
der nationalen Sicherheit und militärischer Angelegenheiten gab. Artikel 100 benennt den Präsidenten als obersten Befehlshaber der Streitkräfte Bulgariens und untersagt die Ernennung eines aktiven Angehörigen der Streitkräfte zum Minister der Verteidigung. Ferner sind in der Verfassung die Rechte und Pflichten des Parlaments geregelt. Diese umfassen unter anderem die Ausrufung des Kriegsrechts und die Mobilmachung der Streitkräfte. Der Einsatz der Streitkräfte kann sowohl der Landesverteidigung gegen ausländische Bedrohung als auch der Durchführung internationaler Verpflichtungen dienen. ${ }^{3}$

Das Gesetz über die Verteidigung und die Streitkräfte der Republik Bulgarien legt ausdrücklich fest, nach welchen Hauptprinzipien und -verfahren die Regierung zur Kontrolle der Streitkräfte vorgehen muss. Das Parlament ist verpflichtet, die parlamentarische Kontrolle auszuüben und die Aktivitäten der Streitkräfte und anderer nationaler Sicherheitsinstitutionen, einschließlich der Abteilung für Staatssicherheit und der Polizei, durch Gesetzgebung und Disziplinarvorschriften zu regeln. Das Gesetz enthält jedoch keine klare Definition der parlamentarischen Aufsicht und sieht nur beschränkte Instrumente zur Durchsetzung der Rechenschaftspflicht und Kontrolle vor.

Allein schon die Existenz eines verfassungsgemäßen und rechtlichen Rahmens der demokratischen Kontrolle der Streitkräfte kann als eine ausreichende Bedingung für die Erfüllung formaler Kriterien angesehen werden. Das Hauptproblem ist nicht das Fehlen rechtlicher Normen, sondern ihre unbefriedigende Umsetzung. Die Begriffe »demokratische Kontrolle«, »zivile Kontrolle«, »demokratische Streitkräfte « und »Demokratisierung des Militärs « werden oft synonym benutzt, ohne eine klare Abgrenzung. Hinsichtlich des rechtlichen Rahmens als Mittel zur Einführung der demokratischen Kontrolle über den nationalen Sicherheitssektor gibt es eine Reihe politischer, sozialer, technischer und sogar kultureller Einschränkungen.

Das Gesetz über die Verteidigung und die Streitkräfte der Republik Bulgarien entspricht im Großen und Ganzen den europäischen Rechtsstandards. Anfang 2004 hat der Ministerrat eine Novelle zu diesem Gesetz beschlossen, die vom Parlament verabschiedet wurde. ${ }^{4}$ Die Änderungen betreffen die Ausstattung des Amtes «Sicherheit - Militärpolizei und Militärischer Abschirmdienst « mit zusätzlichen Vollmachten bei der Beschaffung, Auswertung und Verwendung von nachrichtendienstlichen Informationen. Reglementiert ist ferner die Kontrolle über diese Tätigkeiten und die Möglichkeit zum Informationsaustausch mit anderen Dienststellen und Staatsorganen unter strikter Einhaltung der geltenden gesetzlichen Bestimmungen. Mit den Änderungen wird die Voraussetzung dafür geschaffen, dass Berufsmilitärs Verträge über das Ableisten von Militärdienst außerhalb des Territoriums der Republik Bulgarien abschließen und an internationalen Operationen und Missionen teilnehmen können. Auf diese Weise sind die geltenden Vorschriften an die neuen internationalen Realitäten angepasst, nach denen Bulgarien verpflichtet ist, als Mitglied der internationalen Staatengemeinschaft an den Bemühungen um Frieden und Sicherheit mitzuwirken. Im Jahr

\footnotetext{
3 Art. 84 der bulgarischen Verfassung.
}

4 Gesetzesänderung vom 19.10.2004.
2003 fasste der Ministerrat einen Beschluss zur Durchführung einer strategischen Überprüfung des Zustandes des Verteidigungssektors. ${ }^{5}$ Deren Ziel bestand darin, die Sicherheitsinteressen des Landes und dessen Verteidigungskapazitäten neu zu bewerten. Diese groß angelegte Analyse wurde mit dem Resultat abgeschlossen ${ }^{6}$, dass die geltenden Normen einer grundlegenden Revision unterzogen werden sollen mit dem Ziel, sie an die veränderten Sicherheitsparameter wie auch an die neuen Aufgaben und Verantwortlichkeiten Bulgariens als Vollmitglied der NATO anzupassen.

\section{Parlamentarische Kontrolle}

Die Aufgaben des Parlaments reichen von einer bloßen Überwachungsfunktion gegenüber der Regierung bis hin zur aktiven Mitgestaltung aller Bereiche der Verteidigungspolitik und der militärischen Angelegenheiten. Heute existieren in Bulgarien alle rechtlichen Voraussetzungen für eine wirksame parlamentarische Kontrolle des Sicherheitssektors. In der Praxis sieht sich diese Kontrolle jedoch mit einigen großen Schwierigkeiten konfrontiert.

Das Parlament trifft in der Tat die endgültige Entscheidung über die Zuteilung von Haushaltsmitteln. Doch wenn es um die eigentliche Verwaltung der Finanzmittel für die Verteidigung geht, wird das Bild auch hier wieder etwas unscharf. Es liegt auf der Hand, dass die zivilen und militärischen Beamten des Ministeriums besser über die speziellen Bedürfnisse der Streitkräfte informiert und umfangreicher vorbereitet sind, die finanzielle Ausstattung des Militärs zu definieren. Die Regierung legt dem Parlament das Endprodukt zu einer mehr oder weniger »symbolischen « Genehmigung vor. Die Frage, die sich hier stellt, betrifft das Verhältnis von Effizienz und Legitimität: Erstere sollte nicht auf Kosten der Letzteren durchgesetzt werden. Eine ausgewogene Kombination muss gefunden werden. Die Ursachen für diese Probleme sind fehlende Erfahrungen und Expertise. Der Mangel an Experten und Beratern der Abgeordneten ist ein akutes Problem. Es sei jedoch darauf hingewiesen, dass dieses Problem in vielen jungen demokratischen Staaten auftritt. Es gibt nur eine kleine Zahl von Zivilisten, die über so viel Wissen über militärische Angelegenheiten verfügen, dass sie als zivile verteidigungspolitische Experten eingesetzt werden können.

\section{Kontrolle durch die Exekutive}

Einige Prinzipien können als Minimalanforderungen an die Kontrolle der Streitkräfte durch die Exekutive angesehen werden. Zum einen muss ein klares Unterstellungsverhältnis geschaffen werden, an dessen Spitze zivile Führer stehen. Zum andern sollte zumindest ein Teil des Personals des Ministeriums aus Zivilisten bestehen.

Die Regierung übt die direkte Kontrolle des Militärs über den Minister der Verteidigung aus. Der Minister ist letztendlich 5 Стратегически преглед на отбраната, (Strategische Überprüfung des Zustandes
der Verteidigung), Ministerrats-Beschluss 262/22.04.2003.

6 Ministerrats-Beschluss 465/31.05.2004. 
für die Umsetzung der Verteidigungspolitik, den Aufbau des nationalen Verteidigungssystems und die internationale Kooperation im Bereich der Verteidigung verantwortlich. Die Erhöhung des Anteils der Zivilisten unter den Mitarbeitern des Ministeriums ist jedoch eine Herausforderung für die demokratische Kontrolle des Militärs. Obwohl das Ziel der Personalführungspolitik darin besteht, ein besseres Gleichgewicht zwischen zivilen und militärischen Mitarbeitern zu erreichen, ist die gegenwärtige Personalzusammensetzung im Verhältnis von Zivilisten zu Militärangehörigen nicht zufriedenstellend. Außerdem kann eingewendet werden, dass das Einsetzen von aktiven Angehörigen der Streitkräfte als Leiter wichtiger politischer Abteilungen (beispielsweise der Abteilung für Rüstungspolitik und Technik oder der Abteilung für Verteidigungspolitik) mit den Prinzipien der demokratischen Kontrolle schwer zu vereinbaren sei. Ursache dieser Personalpolitik ist das Fehlen von erfahrenem und qualifiziertem zivilem Personal mit entsprechendem Werdegang. In Zukunft sollte dies jedoch keine Entschuldigung mehr sein, da die Zahl junger ziviler Spezialisten wächst, die an bulgarischen und/oder westlichen Universitäten eine entsprechende Ausbildung erhalten haben und sich allmählich die notwendigen Erfahrungen im nationalen Verteidigungssystem aneignen. Die Politik der Erhöhung des Anteils der Zivilisten unter den Mitarbeitern des Ministeriums muss fortgesetzt werden; damit ist jedoch nicht gesagt, dass professionelle militärische Kenntnisse im Ministerium nicht gebraucht werden - in bestimmten Angelegenheiten sind sie unersetzlich.

Für die demokratische Kontrolle ist es jedoch zwingend geboten, dass verteidigungspolitische Entscheidungen das Prärogativ von Zivilbeamten sind. Eine weitere Ebene der durch die Exekutive ausgeübten Kontrolle der Streitkräfte umfasst den Befehlshaber der Streitkräfte und den Generalstab als das wichtigste militärische Gremium. Obwohl der Grundsatz der Unterordnung des Generalstabes unter den Minister eingehalten wird, können sich in der Praxis doch bestimmte Probleme ergeben. Das betrifft insbesondere die Integration des Generalstabs in das Ministerium. Tatsächlich scheinen diese gelegentlich als zwei getrennte Institutionen zu arbeiten. Einige dieser Probleme sind auf fehlende Koordinierung zurückzuführen. In der Theorie ist der Generalstab dem Befehlshaber unterstellt, der seinerseits dem Minister unterstellt ist. In der Praxis bedeutet dies die Existenz einer zwischengeschalteten Befehlsstufe, die die Wechselbeziehung zwischen dem Ministerium und dem Verteidigungsstab eher komplizierter gestaltet als erleichtert.

\section{Das Verhältnis zwischen Militär und Gesellschaft}

Die Position des Militärs in der Gesellschaft bildet eine der Grundlagen der zivil-militärischen Beziehungen. Mit Hilfe von öffentlichem Informationsmaterial soll das gesellschaftliche Bewusstsein über Verteidigungsfragen im Allgemeinen und die NATO-Mitgliedschaft im Besonderen erhöht werden. Das Ministerium finanziert darüber hinaus spezielle Literatur, wie die Zeitung «Bulgarische Armee «, »NATO und Bulgarien«, sowie andere monatlich erscheinende Zeitschriften.
Kleineren Unfällen in den Streitkräften wird gewöhnlich viel mehr Aufmerksamkeit geschenkt als internationalen Manövern, die auf bulgarischem Boden stattfinden. Dieses Problem ist auf den Mangel an Journalisten mit militärischer Sachkenntnis und den noch nicht ausreichend entwickelten Journalismus in Bulgarien insgesamt zurückzuführen. Trotz ihrer Vorliebe für Sensationsnachrichten und des Fehlens professioneller Kenntnisse auf militärischem Gebiet sind die Massenmedien dem bulgarischen Militär gegenüber positiv eingestellt.

Die Streitkräfte verfolgen ihrerseits eine Politik der »offenen Tür«, indem sie zahlreiche gesellschaftliche Veranstaltungen organisieren. Während dieser Veranstaltungen haben sowohl Militärangehörige als auch Zivilisten Gelegenheit, direkt miteinander ins Gespräch zu kommen.

Ein anderer, weniger sichtbarer, aber sehr wichtiger Bereich der zivil-militärischen Kooperation sind Such- und Rettungsoperationen. Gelegentlich bitten zivile Institutionen die Streitkräfte bei Naturkatastrophen und Unglücken um Hilfe.

Die letzte, aber sicherlich nicht die unbedeutendste Frage der zivil-militärischen Beziehungen ist die Rolle des so genannten dritten Sektors, d.h. der Nichtregierungsorganisationen (NROs). Obwohl die Beteiligung der NROs am Diskurs über die zivil-militärischen Beziehungen und deren Einfluss darauf bisher ziemlich begrenzt waren, nimmt die Bedeutung ihrer inoffiziellen Rolle zu. Eine der prominentesten NROs ist der Bulgarisch-Atlantische Club, der Runde Tische und Konferenzen zu verschiedenen Aspekten der Integration Bulgariens in die NATO organisierte. Meinungsumfragen zeigen, dass das Vertrauen der Öffentlichkeit in das Militär in den letzten zehn Jahren ständig gestiegen ist.

Heute darf das bulgarische Militär nicht am politischen Leben des Landes teilnehmen. So ist es Soldaten untergesagt, Mitglieder in politischen Parteien zu werden. ${ }^{7}$ Es besteht kein Zweifel darüber, dass bestimmte Aktivitäten, die für Zivilisten gestattet sind, der Armee über gesetzliche Regelungen untersagt sein müssen. Die Trennung zwischen Bürger und Soldat ist keine einfache Frage, noch nicht einmal für gefestigte Demokratien. Andererseits impliziert Demokratie als solche den Schutz und die Durchsetzung der Grundrechte von Personen, unabhängig davon, ob sie Zivilisten oder Militärangehörige sind. In diesem Sinn ist die Teilnahme des Militärs am gesellschaftlichen und politischen Leben eine der am heftigsten umstrittenen Fragen, insbesondere bezüglich der Meinungs- und Pressefreiheit. Die Lösung ist abhängig von der Entwicklungsstufe der politischen Kultur und des politischen Bewusstseins einer Zivilgesellschaft. Es wird noch einige Zeit dauern, bis demokratische Normen und Werte im Alltagsleben der bulgarischen Armee verankert sind.

7 Art. 195, Gesetz über die Verteidigung und die Streitkräfte der Republik Bulgarien. 


\section{Ausbildung und Erziehung}

Ziviles Bewusstsein ist eine weitere wichtige Komponente der demokratischen Kontrolle. Die hoch entwickelte Informationsstrategie des Ministeriums hat die Aufgabe übernommen, der Öffentlichkeit Bedürfnisse, Rollen und Aufgaben zu erläutern und dabei auf die demokratischen Methoden abzuheben, die bei ihrem Aufbau und ihrer Tätigkeit genutzt werden. Die Ausbildung und Erziehung des Militärs wie der Zivilisten wurde als entscheidende Voraussetzung für die Schaffung einer funktionierenden demokratischen Kontrolle angesehen. Das Ministerium beteiligt sich an der Schulung von Gruppen von Zivilisten, die auf politischer Seite an der Entwicklung der Streitkräfte mitwirken, wie beispielsweise die »Canadian Military Language School« oder das internationale FellowshipProgramm »Graf Baudissin« (in dessen Mittelpunkt das Werk von Wolf Graf von Baudissin, dem Vater der Inneren Führung der Bundeswehr, steht). Es handelt sich dabei um Mitarbeiter des Ministeriums, Parlamentsabgeordnete und Journalisten.

Auch Medienangehörige erhalten eine gründliche Ausbildung, da auf Verteidigungsfragen spezialisierte Journalisten den Minister auf Reisen im In- und Ausland begleiten. Journalisten wurde zudem die Möglichkeit eröffnet, an Schulungen und Kursen teilzunehmen, die mit NATO-Programmen in Zusammenhang stehen. Darüber hinaus werden ihnen weitere Anreize geboten, ihre Kenntnisse über die Streitkräfte zu vertiefen.

Ein wichtiger Aspekt der demokratischen Kontrolle ist auch die Erziehung von Offizieren. Höhere Offiziere durchliefen eine Ausbildung an westlichen Militärakademien wie dem »U.S. Army War College«. Demokratische Kontrolle und zivilmilitärische Beziehungen waren integraler Bestandteil dieser Ausbildung.

Einige bulgarische Offiziere arbeiteten als Verbindungsoffiziere in verschiedenen NATO-Stäben und brachten Kenntnisse über Entscheidungsprozesse und politische Aufsicht, wie sie in reifen Demokratien praktiziert werden, mit nach Hause. Das Programm »Partnerschaft für den Frieden « und insbesondere sein Planungsteil, der Planungs- und Überprüfungsprozess, waren wichtige Hilfsmittel bei der Gestaltung der demokratischen Kontrolle in Bulgarien. Sie spielten auch eine wichtige Rolle bei der Entmystifizierung der Verteidigung und verdeutlichten die enge Verbindung zwischen den Fähigkeiten des Militärs und der Verwaltung der Mittel, die die Zivilgesellschaft dem Militär zuweist. In diesem Prozess wurden umfangreiche Analysen des Personal- und Ausrüstungsbedarfs erarbeitet, die den Zusammenhang zwischen der Erhöhung der Personalstärke und der Erhöhung der tatsächlichen militärischen Fähigkeit verdeutlichen. Darüber hinaus wurden Transparenz und Rechenschaftspflicht durch die Schaffung eines einheitlichen Buchhaltungssystems verbessert, die es dem Ministerium ermöglicht, alle Bücher des Militärs zu prüfen. Auch der Aufbau eines einheitlichen Planungssystems, das unter der Bezeichnung Planungs-, Programm- und Budgetierungssystem bekannt ist, förderte die nötige Übersicht.

Jede Reform (besonders eine Reform im Verteidigungssektor) kann sensible soziale, beispielsweise Lohn und Beschäftigung betreffende Fragen aufwerfen. Damit die Reform problemlos durchgeführt werden kann, müssen die zivilen Behörden die Bedürfnisse des Militärs verstehen; den Offizieren muss ihrerseits klar sein, dass die politischen Entscheidungen von Zivilisten getroffen werden müssen. Deshalb ist es für die effektive Funktionsweise der demokratischen Kontrolle äußerst wichtig, dass Zivilisten über entsprechende Sachkenntnis verfügen.

\section{Die Zusammenarbeit mit der NATO und die öffentliche Meinung}

Bulgariens erster Beitrag zum Bündnis ist seine strategische Lage: Als Basis für Einsätze auf dem Balkan ist sie ideal. Bulgarien, an der Schnittstelle zum Balkan, dem Kaukasus und dem Mittleren Osten, kommt dabei eine Schlüsselposition zu, welche es gilt, nicht nur als Verbraucher, sondern vielmehr als Garant für Frieden und Sicherheit auszufüllen.

NATO- und EU-Beitritt werden von den Regierungen als »Heimkehr in die europäische Heimat « dargestellt. In den letzten Jahren war Bulgarien bemüht, die »Hausaufgaben « der NATO zu erledigen: Als Mitglied in der Initiative »Partnerschaft für den Frieden « hat sich das Land an den Friedenseinsätzen in Bosnien und Herzegowina und im Kosovo beteiligt. Die Streitkräfte Bulgariens beteiligen sich an den militärischen Missionen in Bosnien, im Kosovo und derzeit auch in Afghanistan. Während des Kosovokrieges hat sich Bulgarien in seinem Verhalten nicht von dem eines tatsächlichen NATO-Mitgliedstaats unterschieden. Auch während der militärischen Operationen der USA und Großbritanniens in Afghanistan hatte der in der Nähe von Burgas (bulgarische Schwarzmeerküste) liegende Militärflughafen eine strategisch wichtige Schlüsselfunktion. Der Stab der internationalen Streitkräfte der Balkanländer in Plovdiv (Bulgarien) sowie die durchgeführten Übungen schaffen für die Zukunft einen realen Beitrag für die Bewältigung von Krisensituationen. Das bedeutet, dass Bulgarien eine Sicherheit »schafft", die nicht nur von der Region selbst konsumiert wird, sondern von ganz Europa.

Neben den sicherheitspolitischen und den militärischen Überlegungen ist ein weiteres Argument von entscheidender Bedeutung: Bulgarien hofft, als NATO-Mitglied in den Beitrittsverhandlungen mit der EU bessere Karten zu haben. ${ }^{8}$ Das gab auch Außenminister Solomon Passy zu verstehen: »Die Einladung der NATO könnte auf natürliche Weise unsere EU-Beitrittsverhandlungen beschleunigen. Die Erfahrungen von Polen, Ungarn und der Tschechischen Republik zeigen, dass sich die wirtschaftliche Lage in diesen Ländern nach dem NATO-Beitritt entscheidend verbessert hat. Vor allem die ausländischen Investitionen sind rapide gewachsen. Die NATO-Mitgliedschaft bringt zahlreiche neue Chancen zur Realisierung nationaler Interessen, und die bevorstehende EUMitgliedschaft wird sogar noch mehr Möglichkeiten bieten. Betrachten wir das Wort Sicherheit im traditionellen Sinn, so sind wir sicherer denn je. Wir verlassen uns im Bereich der Verteidigung nicht nur auf uns alleine, sondern auch auf das

8 Vladislav Todorov, »Die NATO, die bulgarische Frage und deren Apostel«, in: Kultura, Nr. 38, 25.09.98. 
mächtigste und erfolgreichste Bündnis der Welt. Das Potential unserer 25 NATO-Partner übersteigt in überwältigender Weise alles, was wir zu unserer Verteidigung alleine hätten unternehmen können. «9

Allerdings war der bulgarische Beitrag vor dem NATO-Beitritt politisch umstritten. Während des Kosovokrieges ließ sich die bulgarische Regierung auf die reale Gefahr ein, seine westlichen Nachbarn zu provozieren, aber auch die im Oktober 1999 anstehenden Kommunalwahlen zu verlieren. Noch bevor die NATO in dem Konflikt militärisch intervenierte, hatte sich die bulgarische Regierung als erstes Mitglied der »Partnerschaft für den Frieden « bereit erklärt, für die Operation »Joint Guardian« gegen Jugoslawien logistische Hilfe zu leisten. Nach dem Beginn der Bombardierungen verhielt sich die bulgarische Regierung etwas vorsichtiger, indem sie anfangs einen Balanceakt zwischen gutnachbarlichen Beziehungen zu Serbien und NATO-Treue versuchte. Unterdessen hatten sich die innenpolitischen Auswirkungen derart hochgeschaukelt, dass sich die Regierung in offenem Widerspruch zur Mehrheit der bulgarischen Bevölkerung befand. Ungefähr 80 Prozent der Bulgaren lehnten die NATO-Angriffe auf Jugoslawien ab.

War die öffentliche Meinung in Bulgarien noch 1999 eher gegen die NATO eingestellt, so wendete sich das Blatt in den nächsten Jahren radikal: Heute unterstützen über 60 Prozent der Bulgaren die Mitgliedschaft im Bündnis.

\section{Außen- und Verteidigungspolitik - der interna- tionale Kontext}

Die demokratische Kontrolle des Militärs in Bulgarien steht vor zwei Herausforderungen: Sie muss im nationalen Rahmen zum ersten Mal eingeführt und umgesetzt werden und gleichzeitig mit neuen, von der westlichen Sicherheitsgemeinschaft ausgehenden Entwicklungen Schritt halten. Die auf die Kooperation mit der NATO zurückzuführenden Anreize haben eine wichtige Rolle bei der Schaffung des gesetzlichen Rahmens für die demokratische Kontrolle des Verteidigungssektors in Bulgarien gespielt. Es sei jedoch darauf hingewiesen, dass westliche Vorbilder einfach kopiert und direkt übernommen wurden, was mit den unterschiedlichen lokalen Ausprägungen und Besonderheiten der nationalen Verteidigungssysteme manchmal nicht kompatibel ist.

Manchmal wird das Konzept der demokratischen Kontrolle von der Öffentlichkeit oder den Medien als eine von der NATO formulierte Anforderung interpretiert, die ansonsten für den Staat keine Notwendigkeit darstellen würde. Es ist jedoch eine entscheidende Voraussetzung für ein funktionierendes, demokratisches System und die verfassungsgemäße Ordnung, unabhängig von der NATO-Mitgliedschaft. Der internationale Kontext und die Auswirkungen des von außen ausgeübten Drucks dürfen jedoch nicht vernachlässigt werden. Der Prozess der Integration Bulgariens in die EU ist

9 Interview mit Außenminister Solomon Passy für das Magazin »Sicherheitspolitik«, 12/2004. ein weiterer, äußerer Einflussfaktor, der sich positiv auf die Außen- und Verteidigungspolitik auswirken könnte. ${ }^{10}$

Doch auch Bulgarien sieht sich mit der asymmetrischen Bedrohung durch den Terrorismus und der Proliferation von Massenvernichtungswaffen konfrontiert. Diesen Bedrohungen kann nur durch die Zusammenarbeit von Diplomatie, Wirtschaft, Polizei, Nachrichtendienst und, wenn nötig, Militär entgegengewirkt werden. Dabei ist eine unerlässliche Voraussetzung das solidarische und energische Auftreten der internationalen Gemeinschaft. In diesem Zusammenhang ist die Kooperation von NATO und EU von größter Wichtigkeit.

\section{Die Struktur der bulgarischen Armee im Wandel}

Ende der 1980er Jahre hatte Bulgarien noch 167.000 Mann unter Waffen. Das Land war in die Militärstrategie des Warschauer Vertrages eingebunden, die auf eine Konfrontation riesiger Landarmeen auf dem Kontinent ausrichtet war. Ein großes stehendes Heer mit einer Vielzahl von Standorten und einer umfangreichen Reserve prägte die Struktur des Militärs. $\mathrm{Zu}$ diesem Zeitpunkt waren wesentliche Bestandteile einer demokratischen Kontrolle über die Streitkräfte und die Verteidigungspolitik noch nicht entwickelt worden. Es gab keine Einschränkungsmöglichkeit von Seiten des Staates oder der Exekutive hinsichtlich des Einsatzes der bulgarischen Armee. Auch die zivil-militärischen Beziehungen wurden nicht gefördert. Weder eine informierte, öffentliche, »zivilgesellschaftliche« Debatte noch das Recht zur freien Diskussion über den Einsatz von Streitkräften war vorhanden. Gleichzeitig waren die Streitkräfte weitgehend autonom in der Gestaltung der meisten verteidigungspolitischen Inhalte; die Verteidigungsministerien wurden größtenteils mit militärischem Personal ausgestattet, das faktisch wiederum dem jeweiligen Generalstab unterstellt war. Die Errichtung einer demokratischen, zivilen Kontrolle über die Verteidigungspolitik und die militärischen Aspekte der Außenpolitik war keineswegs unproblematisch. Gleichwohl konnten allmählich substantielle Fortschritte bei der Konsolidierung der demokratischen Kontrolle von Streitkräften gemacht werden. Der relative Erfolg bei der Einführung der demokratischen Kontrolle des Militärs durch Zivilisten ist bemerkenswert und augenscheinlich anhand von drei Faktoren zu erklären: Erstens spiegelt er den allgemeinen Trend zur Demokratisierung im Land und die Delegitimierung von Alternativen zur liberalen Demokratie wider. Zweitens ist die demokratische Kontrolle der Streitkräfte Teil des übergeordneten Ziels der Westintegration, das in dem Gedanken der »Rückkehr nach Europa « seinen Ausdruck findet und de facto Bedingung für die Mitgliedschaft in der NATO und in der EU ist. Konkreter noch stellt die NATO mit der Partnerschaft für den Frieden (PfP) den mittel- und osteuropäischen Staaten bei der Reformierung der zivil-militärischen Beziehungen praktische Hilfe und Beratung zur Verfügung. Drittens haben die institutionellen Reformen der 1990er Jahre die demokratische, zivile Kontrolle des Militärs systematisch gefestigt, einen Konsens zugun-

10 Vgl. die Äußerung von Verteidigungsminister Nikolai Svinarov in: Atlantischer Kurier, 07/2004. 
sten demokratischer Modelle zivil-militärischer Beziehungen herbeigeführt und deren Gefährdung durch innenpolitische Veränderungen reduziert.

Die Demokratisierung Bulgariens seit 1989 und die Auflösung des Warschauer Vertrages veränderten die Lage. 1992 wurden das zivile Verteidigungsministerium und der militärische Generalstab der Armee organisatorisch getrennt. Politiker übernahmen nun die Führung der Streitkräfte. Obschon die zivile Kontrolle über die Streitkräfte und das Verteidigungsministerium formal frühzeitig sichergestellt wurde, schritt die Reform des Militärs und der zivil-militärischen Beziehungen in Bulgarien nur langsam voran - einerseits aufgrund des geringen Reformwillens der Regierung, andererseits wegen der Zersplitterung der Opposition. Obgleich die zivile Hoheit ein weitgehend akzeptiertes Prinzip verkörpert, stellen das geringe Engagement des Parlaments sowie die mangelhafte Kontrolle über die dem Verteidigungsministerium unterstehenden Geheimdienste ein Problem dar. Es fehlt überall an unabhängiger ziviler Expertise. ${ }^{11}$

Die Revolution, die derzeit im militärischen Sektor stattfindet, beruht auf komplexen Hightech-Technologien und bevorzugt kleinere, mobilere Streitkräfte. Mit einer schlanken, aber modernen und schlagkräftigen Truppe beteiligt sich das Land an internationalen Einsätzen im Rahmen der Allianz und leistet einen Beitrag zur Stabilität auf dem Balkan. Die Priorität der militärischen Aktivitäten lag seit 1990 vor allem auf internationalen Einsätzen. Soldaten waren an Einsätzen in Kambodscha, Angola, Tadschikistan, Kroatien, Bosnien und Herzegowina, Eritrea und Afghanistan beteiligt. Auch im Irak sind Bulgaren stationiert. Weltweite Einsätze im Verbund mit Partnerstaaten erforderten die Modernisierung der Armee. Um die Streitkräfte mit den zur Verfügung stehenden Ressourcen des Landes auf den neuesten Stand der Technik bringen zu können, mussten sie zunächst verkleinert werden. Bulgarien reduzierte die Friedensstärke auf 45.000 Soldaten. Hinzu kommen etwa 100.000 Reservisten. Damit ging die erste Etappe der Militärreform zu Ende.

Zur Zeit dienen 12.000 Wehrpflichtige. Die Dienstzeit beträgt neun (für Universitätsabsolventen sechs) Monate. Bis 2010 soll jedoch die Umwandlung in eine reine Berufsarmee abgeschlossen sein. ${ }^{12}$ Der Truppenabbau war eine Herausforderung für das ganze Land: Sozialprogramme für die entlassenen Soldaten waren nötig. Auch die Vernichtung überschüssiger Waffen und veralteten Materials kostete viel Geld. Grundlage der Reform war der »Plan $2004 \ll^{13}$, der die Gliederung in Krisenreaktionskräfte, Verteidigungstruppen, Territorialverteidigung und Reserve vorsieht. Hauptziel ist die Entwicklung der Armee zu einer effektiven und einsatzfähigen Truppe, die sowohl zur Landesverteidigung als auch für internationale Friedenseinsätze taugt.

11 Andreas Heinemann-Grüger/Igor Grebenschikov, »Militär und Gesellschaft in Osteuropa «. Die Entwicklung der zivil-militärischen Beziehungen. Reader Sicherheitspolitik, www.reader-sipo.de.

12 Дългосрочна визия за развитие на войските и силите - 2015, (Langfristige Vision für die Entwicklung der Streitkräfte - 2015); Beschluss des Ministerrats vom 29.04.2004.

13 Plan für den Organisationsaufbau des Verteidigungsministeriums und der Streitkräfte bis 2004. http://www.mod.bg.
Ende 2003, ein Jahr früher als geplant, wurden die Schlüsselparameter des Plans 2004 erreicht. Die Infrastrukturen wurden zum Teil abgebaut und einige Waffensysteme ausgemustert. Die Hauptaufgaben zum gegenwärtigen Zeitpunkt bestehen in der Erhöhung des Niveaus der operativen Kompatibilität, insbesondere in denjenigen Einheiten, die an Operationen unter Leitung der NATO teilnehmen sollen. Mit Blick auf die bevorstehende Umwandlung der Streitkräfte in eine Berufsarmee werden immer mehr Berufssoldaten eingestellt. Die vollständige Modernisierung des Militärs dauert noch bis 2015 an. Zunächst stehen der Aufbau eines Luftüberwachungssystems und eines Kommunikations- und Informationssystems für das Heer auf dem Programm. Geleitet von dem Verständnis, dass die NATO-Mitgliedschaft nicht nur geteilte Sicherheit, sondern auch geteilte Verantwortung bedeutet, bereiten sich die bulgarischen Streitkräfte darauf vor, durch die Bereitstellung von Verbänden, die an NATO-Einsätzen teilnehmen sollen, einen Beitrag zur kollektiven Sicherheit und Verteidigung zu leisten. Die bulgarische Armee setzt ihre Beteiligung an den Missionen KFOR, SFOR, ISAF und im Irak mit 820 Militärangehörigen fort. In den letzten zwei Jahren hat sich die Zahl der bulgarischen Militärangehörigen, die an internationalen Missionen teilnehmen, nahezu verzehnfacht. Bulgarien ist sich durchaus der Schwierigkeiten bewusst, die mit der Teilnahme relativ kleiner Einheiten an mehreren Missionen verbunden sind. Seit 1998 erfüllen bulgarische Offiziere die Funktionen auf internationaler Ebene. Bis zum gegenwärtigen Zeitpunkt haben 23 Offiziere verschiedene Posten in den multinationalen NATO-Strukturen bekleidet. Seit November 2003 wurden 16 bulgarische Offiziere in verschiedene NATO-Kommandos und -Stäbe entsandt, die für die Dauer von jeweils drei Monaten konkrete Funktionen in den Strukturen der Allianz erfüllten. Nach der Aufnahme Bulgariens in die NATO erhöhte sich die Zahl dieser Offiziere. ${ }^{14}$

\section{Die Zukunft der demokratischen Kontrolle}

Die Erfahrung zeigt, dass der Weg der Reformen weder leicht noch kurz ist. Es ist wahr, dass die Militärreform in Bulgarien weder abgeschlossen noch rückgängig zu machen ist. Doch haben denn die Reformen in den Streitkräften der anderen Länder das gewünschte Niveau erreicht? Die Zukunft der demokratischen Kontrolle der Streitkräfte in Bulgarien sollte in erster Linie im Zusammenhang mit dem Verständnis für die neuen Aufgaben der demokratischen Kontrolle in der heutigen Welt gesehen werden.

Bulgarien beteiligt sich an internationalen Friedenseinsätzen, also erfolgt die demokratische Kontrolle in diesen Fällen doppelt: Einerseits sind das Parlament und die Regierung darüber in Kenntnis gesetzt, welche konkreten Aufgaben das Militär in einem bestimmten Einsatz erwarten, dessen Organisation oftmals über NATO-Strukturen abgewickelt wird. Andererseits werden die unterschiedlichen Genehmigungen von Seiten der Regierung und des Parlamentes so schnell wie möglich

14 Nikolai Svinarov, Die bulgarische Armee bereitet sich auf den NATO-Beitritt vor, in: Bulgarisches Wirtschaftsblatt und südeuropäischer Report, Sofia, $02 / 2004$. 
erteilt. Dies ist insofern wichtig, als das Tempo vor allem in Konfliktsituationen oft eine entscheidende Rolle spielt. Heute sind diese Entscheidungen oft politischer Art und sollten über politische Kanäle abgewickelt werden. Vor allem in Friedenserhaltungs-Situationen ist ein solides politisches Urteilsvermögen bis hin zum kleinsten Detail erforderlich. Benötigt werden auch politische Berater, die militärisch kompetent und flexibel sind, so dass sie vor Ort in den Konfliktgebieten, wie beispielsweise in Afghanistan, im Kosovo und in Bosnien tätig werden können.

In den unterschiedlichen Ländern des Bündnisses existieren verschiedene Vorschriften für die demokratische Kontrolle der jeweiligen Streitkräfte, deren Regelungen vor dem jeweiligen historischen und kulturellen Hintergrund betrachtet werden müssen. Während des Kalten Krieges waren diese Fragen nicht maßgeblich, da allein die Einsatzbereitschaft der Truppen von Bedeutung war. Glücklicherweise kam es nie zum Ernstfall. Nach dem Ende des Kalten Krieges nahm die Zahl der Einsätze zu. Diese Tatsache erfordert ebenfalls ein realistischeres Herangehen an die Frage des militärischen Befehls und der militärischen Kontrolle sowie der politischen Anleitung und Aufsicht.

\section{Schluss}

Die skizzierten Elemente der Kontrolle der Streitkräfte in Bulgarien machen deutlich, dass es sich bei der Reform des Sicherheitssektors um einen komplexen politischen und ge- sellschaftlichen Prozess handelt, der mit der Installierung von institutionellen Voraussetzungen nicht abgeschlossen ist.

Folgende Grundvoraussetzungen müssen geschaffen werden:

- Der Sicherheitssektor muss gegenüber der zivilen politischen Führung und Zivilgesellschaft rechenschaftspflichtig sein.

- Akteure des Sicherheitssektors müssen in Einklang mit dem Völkerrecht, den demokratischen Grundprinzipien und der Rechtsstaatlichkeit handeln.

- Informationen über Planungen und Budgetfragen sollten für die Politik und die Zivilgesellschaft verfügbar sein.

- Mechanismen zur Stärkung der Transparenz des Sicherheitssektors sollten gefördert und institutionalisiert werden.

- Es sollte ein gesellschaftliches Umfeld geschaffen werden, das die Kontrolle des Sicherheitssektors durch die Zivilgesellschaft begünstigt.

- Die zivilgesellschaftlichen Vereinigungen, die sich mit Fragen des Sicherheitssektors befassen, sollten gefördert werden.

- Es sollte ein regionales Umfeld geschaffen werden, das sich nachhaltig mit den Folgen von bewaffneten Konflikten und autoritärer Herrschaft befasst, z.B. mit Fragen der Abrüstung, der Demobilisierung, der Reintegration von Kombattanten, etc. $« 15$

15 Hans-Georg Ehrhart, Das »Internationale Fellowship-Programm Graf Baudissin « am Institut für Friedensforschung und Sicherheitspolitik und der »Export « der Inneren Führung in die Armeen postkommunistischer Staaten: Erfahrungen, Herausforderungen, Defizite und Ausblick, in: Martin Kutz (Hrsg.): Gesellschaft, Militär, Krieg und Frieden im Denken von Wolf Graf von Baudissin, Baden-Baden, 2004, S.171. 